\title{
Neurotransmitter Storage and Recycling: Two Transporter Families Govern Complementary Synaptic Roles with Vast Medical Applications and Therapeutic Perspectives: A Commentary
}

\author{
Elías Leiva and J Pablo Huidobro-Toro* \\ Department of Biology, University of Santiago, Chile
}

Submission: April 04, 2017; Published: June 19, 2017

*Corresponding author: J Pablo Huidobro-Toro, Center for the Development of Nanosciences and Nanotechnology (CEDENNA), Nucleotide Laboratory and Nano Neurobiology Laboratory, Department of Biology, Faculty of Chemistry and Biology, University of Santiago, Alameda 3363, Estación Central, Santiago, Chile, Tel: 56-2-22718-1144; Email: juan.garcia-huidobro@usach.cl

Keywords: Neurotransmitter transporters; SLC17 family members; SLC18 family members; NET vs vMAT; GluTvs vGLUT; DAT

\section{Commentary}

The storage of neurotransmitters in synaptic vesicles and its recycling, either intact or as a precursor molecule after its release, are two key concepts underlying the synaptic physiology that apply to both central and peripheral neurons. Although within the past 50 years we have vastly increased our understanding of the neurochemistry supporting transmitter synthesis, and the cytoarchitectural features of the nerve ending varicosities where these events take place, the cellular and molecular principles that govern transmitter storage and recycling are still elusive in many respects. We teach that transmitter storage and its reutilization are two separate events, causally related and depend on two distinct types of transporter proteins. One dependent of transporters recycles the transmitter back to the nerve terminal (reuptake) once it is released and in a second independent step, another transporter accumulates the neurotransmitter in the synaptic vesicles for its subsequent release. The first step is spatially restricted to the neuron plasma membrane in the varicosities, while the second step is exclusively localized in the intracellular space, in the membranes of the synaptic vesicles. Moreover, several evidences indicate that neurotransmitter accumulation in the synaptic vesicles and neurotransmitter reuptake occurs through the set of functional and structural non-related transporter proteins (Figure 1), furthermore, these transporters are encoded by separate genes. Why these two types of transporters for a same neurotransmitter molecule evolved, and what is the physiologic advantage of two-separate mechanisms for neurotransmitter transport in the synaptic dynamics and its continuous changes in response to environmental challenges?

The first evidence of these two mechanisms emerged from pharmacologists and was based on the use of the first antidepressant agents and CNS stimulants, which specifically target plasma membrane transporters rather than the vesicular transporters. It is well recognized that tricyclic antidepressants inhibit NET (norepinephrine transporter) and SERT (serotonin transporter) while cocaine inhibits DAT (dopamine transporter) increasing the neurotransmitter levels in the synaptic cleft. Solid evidence of the function of these transporters came from cell biologists and geneticists, who demonstrated that deletion of these genes (NET, SERT and DAT) decreases monoamine reuptake. The drugs targeting these plasma membrane transporters exert major clinical effects and are widely used in psychiatry and in chronic pain management; cocaine is an addictive central stimulant that is a prototype model for drug dependency studies.

Moreover, neurochemical experiments using reserpine or tetrabenazine allowed differentiating between recycling from vesicular transporter process. These drugs inhibit noradrenaline and dopamine vesicular accumulation and result in a blockage of sympathetic reflexes or pseudo Parkinsonism. Reserpine was 
discovered years later as a target of the vesicular monoamines transporter (vMAT) with no substantial effect in the reuptake transporters. Because of the inhibition of the sympathetic reflexes, this drug was the first massive antihypertensive during the late $50 \mathrm{~s}$ and $60 \mathrm{~s}$, but it was soon replaced by $\beta$-adrenergic blockers, which were the dominant drugs used as universal antihypertensive agents for more than two decades. Because of the numerous and serious side effects of reserpine, nowadays, this effective drug is largely out of medical use [1-8].

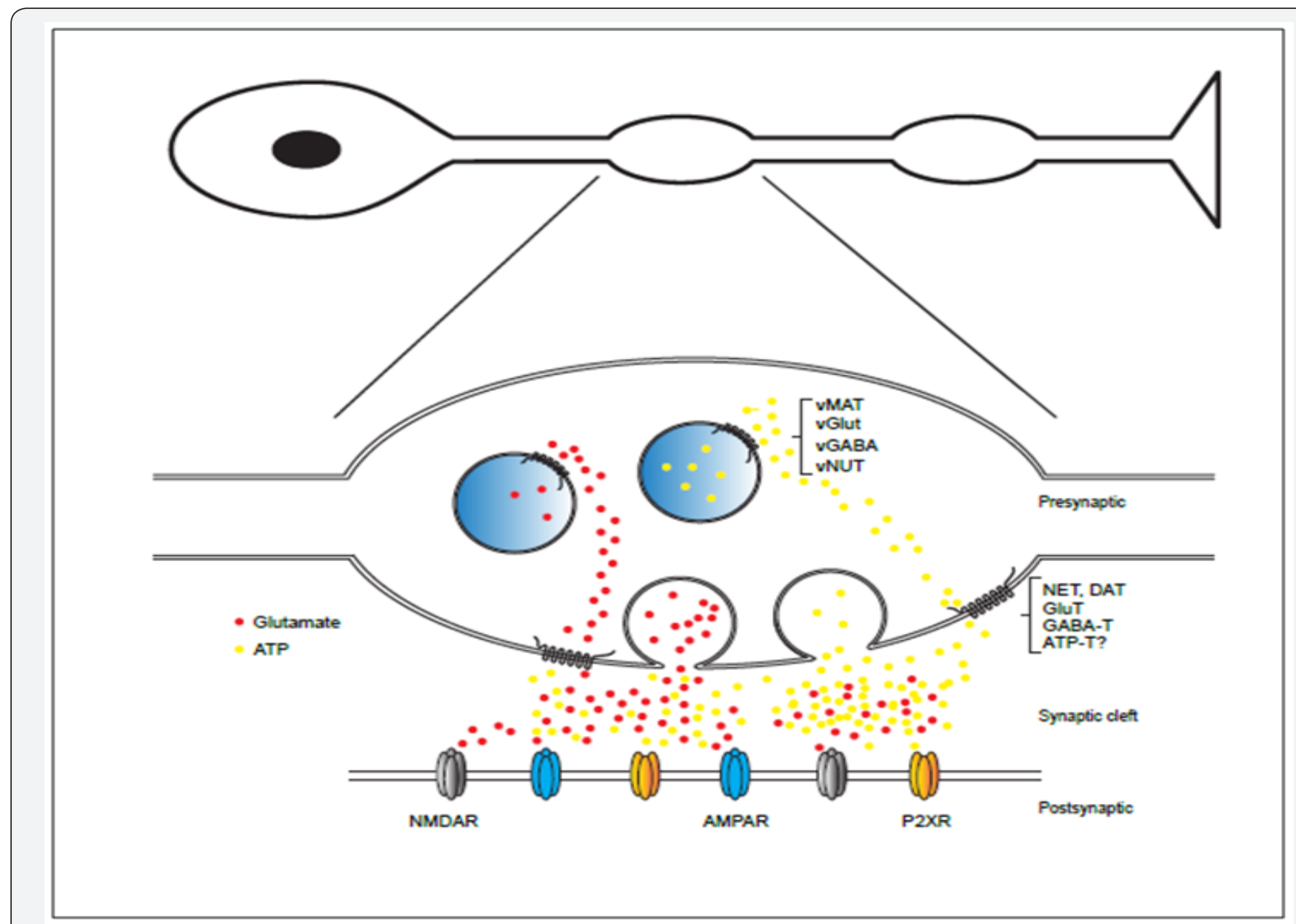

Figure 1: Schematic cartoon of a neuronal varicosity showing presynaptic and postsynaptic elements. Synaptic varicosity illustrates the corelease of glutamate and ATP by a CNS neuron. Two transporters are illustrated in the dynamics of a nerve ending; both are single proteins of 12 transmembrane domains each anatomically localized in different cell membranes. One of these transporters, localized in the plasma membrane of the nerve terminal recycles back (reuptake) to the nerve terminal the neurotransmitter recently released and shows specificity for different substrates. NET and DAT refer to a monoamine transporter with selectivity for noradrenaline and dopamine, respectively. GluT, GABAT are selective transporters for glutamic acid and GABA, ATP-T is the putative ATP transporter. In contrast to these proteins, another set of transporters localized exclusively in the membrane of the synaptic vesicle incorporates the transmitter into the synaptic vesicle. Such is the case of VMAT (vesicular monoamine transporter) or vGlut (vesicular glutamate), while VGABA and vNUT depict vesicular transporters for GABA and nucleotides, respectively.

Genome sequencing allowed identifying the different genes encoding the reuptake and the vesicular transporters (Figure 1). For instance, two families of genes encode the glutamate transporters, which comprise 5 genes for the reuptake transporters (SLC1A3, SLC1A2, SLC1A1, SLC1A6 and SLC1A7) and 3 genes for the family of vesicular transporters (SLC17A7, SLC17A6 and SLC17A8). Key differences between these families are related to their higher gglutamate, affinity being the vesicular transporters 100 to 1000 times lower than the recycling transporters; in addition the vesicular transporter depends on $\mathrm{H}^{+}$ATPase, which generates the electrochemical gradient necessary for neurotransmitter vesicular loading.
Later studies identified 3 vesicular glutamate transporters and clarified that these proteins share similarities with phosphate and anionic transporters, all of them classified as members of the SLC17 protein family, which encodes 9 transporters, including 3 vesicular glutamate transporters (vGLUT1-3), a glutamate/aspartate transporter (vEAT, referring to excitatory amino acid transporter) and a vesicular nucleotide transporter (vNUT, SLC17A9) among others.

Recently, data has accumulated in favor of a novel vesicular nucleotide transporter (vNUT), which might participate in the accumulation of ATP inside the synaptic vesicles together 
with other neurotransmitter such as noradrenaline and/or acetylcholine. Additionally, 4 genes encode for the inorganic phosphate transporters, these are abundantly expressed in kidney and liver. The members of this family share both structural and functional characteristics, they are all based on putative twelve transmembrane domains, show chloride- and proton gradient dependent transport, all are blocked by azo dyes such as Evans blue and its structural analogues. Although highly expressed in the synaptic vesicles of the nervous system, the phosphate transporters are known to be expressed in the cell membrane of excreting organs and serve to eliminate negatively charged metabolites, anions or xenobiotics, for example, p-aminohippuric acid excretion, a molecule widely used in the past for human renal clearance measurements. Furthermore, SLC17 family share structural features with the SLC18 family of transporters, which accumulate positively charged molecules inside the synaptic vesicle such as monoamines (vMAT1 and 2), acetylcholine/choline (vACh), and the recently identified polyamines transporter (vPAT, for spermine and spermidine). vMAT 1 and 2 were previously known because of their significance in the effects of reserpine and for their implications in neuro-affective disorders as well as Parkinson's disease. Finally, a new family of these molecules was cloned and identified as SLC32, which comprise one protein that transports GABA and glycine into neuronal vesicles in nerve terminals. Overall, the identification of the vesicular transporters gives account for the neuronal vesicular storage mechanism of the most important charged neurotransmitters.

Other neurotransmitter/modulator such as adenosine has only a recycling membrane transporter, but not their vesicular counterparts. The inverse holds true for ATP, while vNUT accounts for ATP vesicular storage, we ignore whether ATP is recycled as itself or as adenosine, an ATP metabolite generated by ecto ATPases. Moreover, other signaling molecules such as eicosanoid metabolites or gasotransmitters (NO, CO or $\mathrm{H}_{2} \mathrm{~S}$ ) appears not to be stored in vesicles, but delivered upon tissue synthesis and demand. Furthermore, peptide transmitters like hormones or modulators such oxytocin/vasopressin, CRF, ACTH, TSH or $\beta$-endorphin among others, are released from larger precursor proteins through proteolytic cleavage mediated by enzymes, along with the vesicle trafficking from the soma to the nerve ending varicosities.

An unresolved phylogenetic question refers to the requirement of different transporters and their genes for recycling and vesicular storage. At present, we ignore the exact evolutionary pressure that guided and determined their biological significance. This dual set of transmitter transporters might have enriched the synapse with the dynamic mechanism required for its functional bases and provided independent check-points to ensure synaptic fidelity. For instance, the transmitter reuptake must reduce the transmitter concentration in the synaptic cleft in matters of milliseconds to avoid receptor desensitization or even toxic effects such as glutamate excitotoxicity. Therefore, these transporters must operate at fast rate in the peak of the synaptic transmitter release, since their main role is apparently to end receptor activation at the synapse. In contrast, the vesicular transmitters operate continually, likely at a constant rate, to ensure the speed of synaptic vesicle loading. We infer that the kinetics, and the transmitter concentration gradient at which they operate, imposed a strong evolutionary pressure to establish complementary roles for these two sets of transporter molecules and their genes resulting in high transmitter competence.

Multiple excitatory and inhibitory synapses take the advantage of this double control mechanism/process, including glio-transmission interactions. As it was exemplified, both excitatory and inhibitory neurons, either from central and/or peripheral synapses, are endowed with this dual mechanism of transmitter recycling and storage, further supporting our contention of their efficient role in synaptic transmission functioning. An additional comment based on our teaching experience indicates that undergraduate and medical students always misunderstand this double transporter mechanism and simplify presynaptic transporter proteins to a single entity rather than acknowledging their distinctive and functionally segregated physiological roles. Despite this complexity, targeting these transporters is a certain mechanism for drug development and fostering unforeseen applications in experimental neurosciences as well as in clinical practice. In view of the critical role of these transporter proteins for the synapse, it is our view that tailor-made drugs blocking selectively the activity of these transporters may provide therapeutic opportunities of clinical relevance, for example as a novel approach in Parkinson's disease, pain management and/or glucose homeostasis, among other conditions.

\section{Conclusion}

In summary, this commentary highlights the notion that neurotransmitter transporters are essential synaptic constituents allowing separate regulatory mechanisms, which evolved and became widespread for excitatory and inhibitory neurons in the brain, enteric nervous system and other peripheral neurons. The evolutionary strategy of transmitter recycling poses a beneficial advantage reassuring the quick reposition of a crucial neuronal element for fast and continue cell communication. These proteins are target of numerous drugs with recognized clinical relevance for neurology and psychiatry.

\section{Acknowledgement}

Funded in part by FONDECYT 11140731 and PAI 791440059 to EL and FONDECYT 117-0842 to JPHT; CEDENNA grant FB0807 provided additional funds.

\section{References}

1. Bellochio EE, Reimer RJ, Fremeau RT, Edwards RH (2000) Uptake of glutamate into synaptic vesicles by an inorganic phosphate transporter. Science 289(5481): 957-960. 
2. Chaudhry FA, Boulland JL, Jenstad M, Bredahl MK, Edwards RH (2008) Pharmacology of neurotransmitter transport into secretory vesicles. Handb Exp Pharmacol 184: 77-106.

3. El Mestikawy S, Wallén-Mackenzie A, Fortin GM, Descarries L, Trudeau LE (2011) From glutamate co-release to vesicular synergy: vesicular glutamate transporters. Nat Rev Neurosci 12(4): 204-216.

4. Omote H, Miyaji T, Hiasi M, Juge N, Moriyama Y (2016) Structure Function and drug interactions of neurotransmitter transporters in the postgenomic era. Annu Rev Pharmacol Toxicol 56: 385-402.

5. Blakely RD, Edwards RH (2012) Vesicular and Plasma Membrane Transporters for Neurotransmitters. Cold Spring Harb Perspect Biol 4(2): a005595.

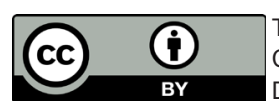

This work is licensed under Creative Commons Attribution 4.0 Licens

DOI: 10.19080/OAJNN.2017.04.555636
6. Reimer RJ (2016) SLC17: a functionally diverse family of organic anion transporters. Mol Aspects Med 34(2-3): 350-359.

7. Rusell JT (1984) $\Delta \mathrm{pH}, \mathrm{H}$ diffusion potentials, and $\mathrm{Mg}^{2+}$ ATPase in neurosecretory vesicles isolated from bovine neurophypophyses. J Biol Chem 259(15): 9496-9507.

8. Yasushi Shigeri, Rebecca P Seal, Keiko Shimamoto (2004) Molecular pharmacology of glutamate transporters, EAATs and VGLUTs. Brain Res Brain Res Rev 45(3): 250-265.

Your next submission with Juniper Publishers will reach you the below assets

- Quality Editorial service

- Swift Peer Review

- Reprints availability

- E-prints Service

- Manuscript Podcast for convenient understanding

- Global attainment for your research

- Manuscript accessibility in different formats

( Pdf, E-pub, Full Text, Audio)

- Unceasing customer service

Track the below URL for one-step submission https://juniperpublishers.com/online-submission.php 